\title{
The g13 Experiment at Jefferson Lab: Strangeness Production on the Neutron in the Deuteron with Polarized Photons: $\vec{\gamma} n \rightarrow K \vec{Y}$
}

\author{
E. Munevar*, B. L. Berman*, P. Nadel-Turoński*,†, and the CLAS \\ Collaboration $^{\dagger}$
}

*The George Washington University, Washington, DC 20052

$\dagger$ Thomas Jefferson National Accelerator Facility, Newport News, Virginia 23606

\begin{abstract}
Strangeness has been shown to be important for the understanding of the so-called missing resonances. Due to the scarce experimental data in strangeness photoproduction on the neutron, phenomenological models such as coupled-channels analyses resort to certain approximations that do not allow getting either accuracy or agreement between different analyses when extracting resonance parameters. Thus, in order to obtain high-quality data on the neutron channels, a new experiment (designated g13), based on a liquid deuterium target and a polarized photon beam (both circular and linear polarization) covering from threshold to $2.3 \mathrm{GeV}$ has been done at the Thomas Jefferson National Accelerator Facility. In this paper, a brief description of the g13 experiment is given.
\end{abstract}

Keywords: Kaon photoproduction, missing resonances, polarization observables

\section{INTRODUCTION}

The strong-interaction mechanism at the level of hadronic physics is still not well understood. Experimental measurements are well reproduced by quantum chromodynamics (QCD) at high energies, but its perturbative calculations diverge at intermediate energies. The difficulties in solving QCD in this intermediate-energy region makes it necessary for us to resort to phenomenological models.

Standard quark effective-potential models, in which the nucleon is represented by three degrees of freedom, predict a richer resonance spectrum than that measured experimentally $[1,2]$. As opposed to this model, such a rich spectrum is not seen for the case of quark models with fewer degrees of freedom, such as those based on quarkdiquark interactions inside the nucleon, the so-called diquark models [3]. Therefore, there is a set of excited states for the nucleon not yet found experimentally but predicted theoretically by the SU(6) symmetric constituent quark model; these hidden states are known as the "missing resonances." Finding some of these states experimentally would confirm the validity of the quark-quark interaction model and, at the same time, would call into question the existence of strongly correlated quark pairs inside the nucleon. This is precisely the main goal of the g13 experiment: to search for missing resonances on the nucleon by using a polarized-photon beam and determining not only the cross section but also most of the polarization observables associated with the corresponding strange-particle reaction channels.

CP947, VII Latin American Symposium on Nuclear Physics and Applications

edited by R. Alarcon, P. L. Cole, C. Djalali, and F. Umeres

(C) 2007 American Institute of Physics 978-0-7354-0461-8/07/\$23.00 


\section{STRANGENESS PHOTOPRODUCTION}

Most of the experimentally known resonances come from channels that include pions either in the initial state or in the final state of the reaction (the other known resonances are related to $\eta$ and $\omega$ production). The question is where to look for the missing resonances. Theoretical calculations have shown that missing resonances could be associated with resonances that couple weakly to pion channels and possibly strongly to photon channels [4]; this means that strangeness channels might be a good place to perform such a search. Specifically, the $K \Lambda$ and $K \Sigma$ photoproduction channels are the most important channels to look for these missing excited states of the nucleon. Recent theoretical analyses [5, 6] of the same CLAS polarization data on the proton [7] result in claims of evidence either for a $\mathrm{P}_{13}$ or a $\mathrm{D}_{13}$ resonance at $1900 \mathrm{MeV}$. Data for strangeness photoproduction on the neutron is needed to tell which theoretical analysis, if either, is correct.

Nucleon resonances can be studied by using phenomenological models that extract resonance parameters, including mass, total width, and branching ratios. These models use Feynman diagrams to include contributions in the reaction from Born terms, from nucleon resonances, and from non-resonant corrections; the vertices of these diagrams are expressed in terms of parameters to be fitted to the experimental data. In the missing-resonance energy region $(>1.7 \mathrm{GeV})$ there are several open channels which make it necessary to implement in the models not only purely hadronic data, but also photoproduction data. The simultaneous inclusion of both meson-nucleon and photonnucleon reactions in a single phenomenological model constitutes what is known as "coupled-channels analysis"1 $[8,9]$. Recent calculations based on the coupled-channels approach have shown the absolute necessity of having experimental data for the strangeness photoproduction channels on the neutron [10]. Reliable results from coupled-channels analyses currently require data for the $\gamma n \rightarrow K^{0} \Lambda^{0}, \gamma n \rightarrow K^{0} \Sigma^{0}$, and $\gamma n \rightarrow K^{+} \Sigma^{-}$reactions. These reactions therefore represent a very important input for coupled-channels analyses either for ultimately discovering unseen nucleon resonances or for ruling out their existence [11].

\section{THE g13 EXPERIMENT}

In order to search for missing resonances, we have proposed and carried out a new experiment [12] designed to produce strangeness data on the neutron using polarized photons with very high statistics. This experiment has finished its run recently at the Thomas Jefferson National Accelerator Facility, using the CLAS detector system together with the polarized tagged-photon beam in Hall B. The experiment (consisting of two parts and designated as g13) is intended to fulfil the conditions for experimental data required by the coupled-channels approaches: high-quality data with good kinematic coverage

\footnotetext{
${ }^{1}$ The main point of a coupled-channels analysis is to look for the same resonance in different open channels by fitting all the data simultaneously and in a consistent way. The nonresonant backgrounds and the interferences with other states associated with each open channel can be different.
} 
and many experimental observables available for each reaction channel. Thus, the experiment makes use of a liquid deuterium target $L D_{2}$ and both circularly (g13a) and linearly (g13b) polarized photon beams with the measurement of kaons or their decay products (pions) and the decay products of the $\Lambda$ and $\Sigma$ (pions and nucleons) in the final state. The first part of the experiment used circularly polarized photons in the photon energy range up to $2.5 \mathrm{GeV}$, and was run in the Fall of 2006. The second part used linearly polarized photons ranging from 1.1 to $2.3 \mathrm{GeV}$, and was run in Spring-Summer 2007. The polarization observables obtained from both parts add up to seven: hyperon recoil polarization $(P)$, beam $(\Sigma)$ and target $(T)$ asymmetries, and circular and linear polarization-transfer coefficients $\left(C_{x}, C_{z}, O_{x}\right.$, and $\left.O_{z}\right)$. Adding to these observables the unpolarized differential cross section $(d \sigma / d \Omega)$, we obtain a total of eight observables (out of ten independent observables) for each channel. The two remaining observables require the use of a polarized target [12].

For $g 13 \mathrm{a}$, Fig. 1 depicts the invariant mass of $\mathrm{p} \pi^{-}$and $\pi^{+} \pi^{-}$pairs which show clearly the lambda and kaon peaks for the reaction $\gamma n \rightarrow K^{0} \Lambda$. Plots are based on 15 million triggers with a 3 -pass electron beam of $1.9 \mathrm{GeV}$ and $84 \%$ polarization. We expect to have about $2 \times 10^{5}$ exclusive $K^{0} \Lambda$ events.
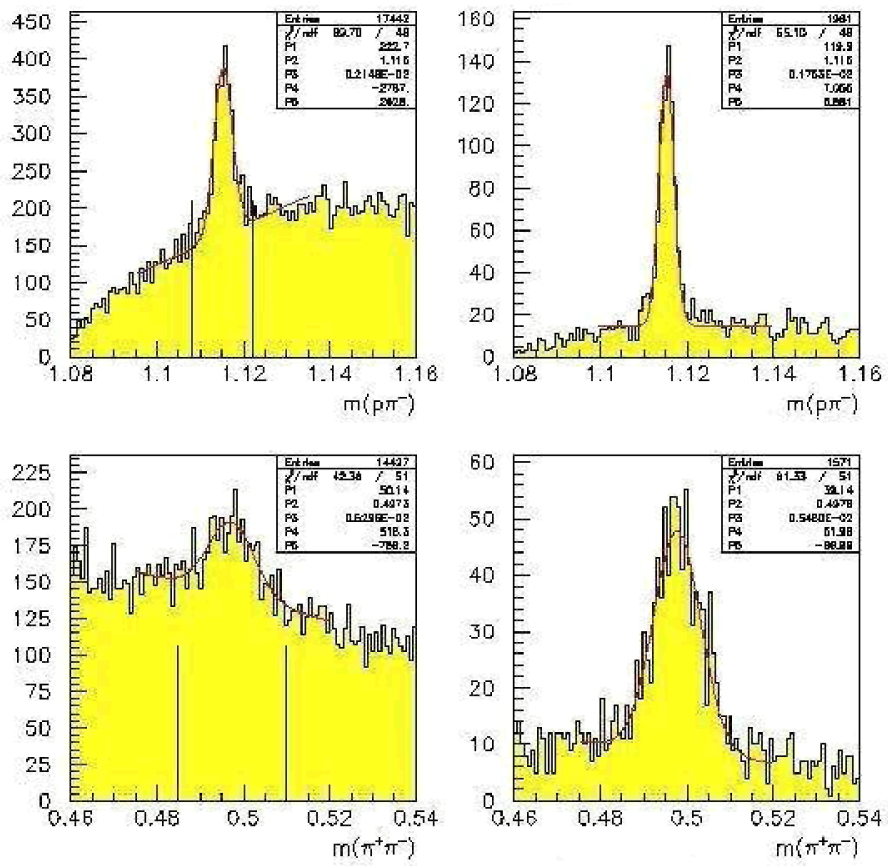

FIGURE 1. Invariant masses of $\mathrm{p} \pi^{-}$and $\pi^{+} \pi^{-}$. The top right plot shows a cut on the kaon mass between 0.485 and $0.510 \mathrm{GeV} / \mathrm{c}^{2}$. A cut on the mass of lambda $1.108<\mathrm{m}_{\wedge}<1.122 \mathrm{GeV} / \mathrm{c}^{2}$ is used in the botton right plot. The data in the figures represent only a tiny fraction $(<0.1 \%)$ of our total data.

As shown in Fig. 2, the quality of data in g13 can also be seen from the 13 b run by looking at the coherent bremsstrahlung spectrum (left plot) and the azimuthal asymmetry 
(right plot) for events with only two particles in the final state (mainly, $\gamma n \rightarrow \mathrm{p} \pi^{-}$).
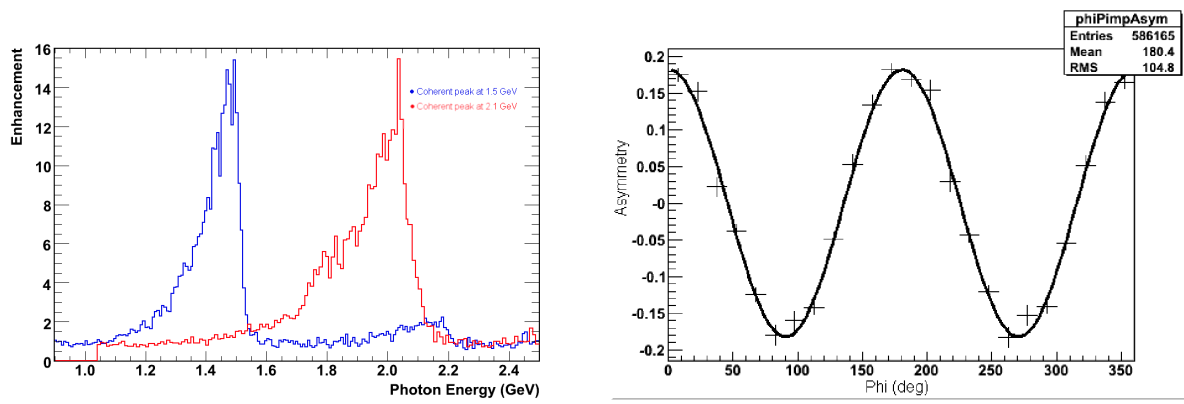

FIGURE 2. The left plot represents the coherent spectrum with edges at $1.5 \mathrm{GeV}$ (blue line) and 2.1 $\mathrm{GeV}$ (red line). The right plot shows the azimuthal asymmetry for events with only two particles back to back in the start counter for $1.3<E_{\gamma}<1.5 \mathrm{GeV}$.

\section{CONCLUSIONS}

In g13 we obtained about 50 billion triggers. Out of these, about half a million correspond to good strangeness-production events divided roughly half and half between circular and linear polarization. As can be seen from the figures shown above, we have obtained a data set of high statistics and quality in the g13 experiment.

\section{ACKNOWLEDGMENTS}

This work was supported by the U.S. Department of Energy under Grant No. DE-FG0295ER40901.

\section{REFERENCES}

1. S. Capstick and N. Isgur, Phys. Rev. D 34, 2809 (1986).

2. S. Capstick and W. Roberts, Phys. Rev. D 49, 4570 (1994).

3. E. Santopinto, Phys. Rev. C 72, 022201 (2005).

4. S. Capstick and W. Roberts, Phys. Rev. D 58, 074011 (1998).

5. V. A. Nikonov et al., arXiv: 0707.3600v1 (2007).

6. C. Bennhold et al., nucl-th/0008024 (2000).

7. R. Bradford et al., Phys. Rev. C 75, 035205 (2007).

8. G. Penner and U. Mosel, Phys. Rev. C 66, 055211 (2002).

9. V. Shkylar et al., Phys. Rev. C 72, 015210 (2005).

10. A. B. Waluyo, Baryon Excitation through Meson Hadro- and Photoproduction in a CoupledChannels Framework, Ph.D. thesis, The George Washington University (2006).

11. I. S. Barker et al., Nucl. Phys. B95, 347 (1975).

12. P. Nadel-Turoński et al., Kaon Production on the Deuteron Using Polarized Photons, PAC30 Proposal: PR-06-114, 2006. 